Article

\title{
Integrated Approaches to Reveal Genes Crucial for Tannin Degradation in Aureobasidium melanogenum T9
}

\author{
Lin-Lin Zhang ${ }^{1,+}$, Jie Li ${ }^{2,+}$, Yi-Lin Wang ${ }^{3}$, Song Liu ${ }^{4}$, Zhi-Peng Wang ${ }^{5, *}$ and Xin-Jun Yu ${ }^{6}$ \\ 1 College of Chemistry \& Environmental Engineering, Shandong University of Science \& Technology, \\ Qingdao 266510, China \\ 2 Laboratory for Marine Fisheries and Aquaculture-Qingdao National Laboratory for Marine Science and \\ Technology, Yellow Sea Fisheries Research Institute, Chinese Academy of Fishery Sciences, \\ Qingdao 266071, China \\ 3 College of Science, China University of Petroleum, Qingdao 266580, China \\ 4 Development \& Reform Bureau, West Coast New Area, Qingdao 266000, China \\ 5 Key Laboratory of Sustainable Development of Polar Fishery, Ministry of Agriculture and Rural Affairs, \\ Yellow Sea Fisheries Research Institute, Chinese Academy of Fishery Sciences, Qingdao 266071, China \\ 6 Key Laboratory of Bioorganic Synthesis of Zhejiang Province, College of Biotechnology and Bioengineering, \\ Zhejiang University of Technology, Hangzhou 310014, China \\ * Correspondence: spirit87@163.com; Tel.: +86-135-8926-0092; Fax: +86-532-8584-1193 \\ + These authors contributed equally to this work.
}

Received: 22 July 2019; Accepted: 27 August 2019; Published: 2 September 2019

check for updates

\begin{abstract}
Tannins biodegradation by a microorganism is one of the most efficient ways to produce bioproducts of high value. However, the mechanism of tannins biodegradation by yeast has been little explored. In this study, Aureobasidium melanogenum T9 isolated from red wine starter showed the ability for tannins degradation and had its highest biomass when the initial tannic acid concentration was $20 \mathrm{~g} / \mathrm{L}$. Furthermore, the genes involved in the tannin degradation process were analyzed. Genes $\tan A, \tan B$ and $\tan C$ encoding three different tannases respectively were identified in the A. melanogenum T9. Among these genes, $\tan A$ and $\tan B$ can be induced by tannin acid simultaneously at both gene transcription and protein expression levels. Our assay result showed that the deletion of $\tan A$ and $\tan B$ resulted in tannase activity decline with $51.3 \pm 4.1$ and $64.1 \pm 1.9 \mathrm{U} / \mathrm{mL}$, respectively, which is much lower than that of $A$. melanogenum T9 with $91.3 \pm 5.8 \mathrm{U} / \mathrm{mL}$. In addition, another gene coding gallic acid decarboxylase ( $\mathrm{gad}$ ) was knocked out to better clarify its function. Mutant $\Delta$ gad completely lost gallic acid decarboxylase activity and no pyrogallic acid was seen during the entire cultivation process, confirming that there was a sole gene encoding decarboxylase in the A. melanogenum T9. These results demonstrated that $\tan A, \tan B$ and gad were crucial for tannin degradation and provided new insights for the mechanism of tannins biodegradation by yeast. This finding showed that $A$. melanogenum has potential in the production of tannase and metabolites, such as gall acid and pyrogallol.
\end{abstract}

Keywords: tannins biodegradation; Aureobasidium melanogenum; Tannase; gallic acid decarboxylase

\section{Introduction}

Aureobasidium genus is popularly known as black yeast due to its melanin production and is frequently encountered in soil, water, the phylloplane, wood, and many other plant materials [1,2]. Their ubiquitous distribution in diverse environmental conditions makes them an easily accessible source for biotechnological and environmental applications [3]. Thus, A. pullulans has been regarded 
as a safe and important biotechnological yeast for bioproducts' production, like poly ( $\beta$-L-malic acid), heavy-oil liamocins, amylase, proteinase, lipase, pullulan, and siderophore [1,4-8].

Tannins are the second most abundant group of phenols in nature, and their molecular weight depends on the bonds possessed with proteins and polysaccharides [9]. Nevertheless, owing to the ability to form strong complexes with different minerals and macromolecules, tannins not only aggregate the precipitates in the beverages but also raise serious environmental pollution problems [9-11]. In addition, tannins negatively affect the nutritional quality of the feed and significantly reduce the intake by livestock because of their bitter taste $[12,13]$.

These undesirable effects of tannins can be reduced or eliminated by certain microorganisms or enzymes. The degradation pathway of hydrolyzable tannins, like gallotannins, has been well understood in bacterial and fungal systems. In the fungal strain, the first step is degradation of tannic acid to form glucose and gallic acid which is converted to pyrogallol by gallic acid decarboxylase. Later, pyrogallol is converted to hydroxymuconic acid by pyrogallol dioxygenase, and then is converted to pyruvate. Pyruvate is finally metabolized through tricarboxylic acid cycle [13]. However, compared to bacteria and fungi, only a few genera in yeast, like Arxula adeninivorans, Candida utilis, Debaryomyces hansenii, and Mycotorula japonica, were observed to degrade the tannins, and little is known about their tannin-degrading pathways [12,14-16]. To the best of our knowledge, A. melanogenum T9 was the first strain to be identified as capable of tannin degrading in the Aureobasidium genus.

Tannase is one of the most studied enzymes in tannins biodegradation, and it can break the ester bonds present in gallotannins, ellagitannins, complex tannins, and gallic acid esters. Tannase have some important applications in various industries and commercially available tannases have mainly been produced by the fermentation of fungi up to now, especially the Aspergillus species [17]. Many strains of $A$. pullulans also harbor a wide range of industrially important enzymes due to their ubiquitous occurrence, biochemical diversity and easily accessible features. Therefore, tannase production by Aureobasidium genus might constitute an adequate alternative to fungal enzymes [3]. It has been found that several different tannases coexist in the fungal or yeast strain, like Colletotrichum graminicola M1.001, Aspergillus niger CBS513.88 and Aureobasidium melanogenum CBS 110374 [18]. Up to now, the strongest reviews regarding tannases were focused on industrial applications and patentability; the respective roles of these genes in tannin catabolism has not been analyzed.

Gallic acid decarboxylase is a kind of nonoxidative aromatic acid decarboxylase and induced by tannins or gallic acid. It is responsible for the decarboxylation of gallic acid to pyrogallol, the second step in the degradation of polyphenol tannic acid. This phenol derivate pyrogallol also has several important industrial applications, for example, in staining of leathers, developing agent in photography, coloring of hair, and cosmetic products [19,20]. Recently, the function of gene AGDC1 coding gallic acid decarboxylase was studied in order to elucidate its role in tannic acid degradation [14]. Interestingly, gene $A G D C 1$ deletion leads to growth inhibition of $A$. adeninivorans when gallic acid is present in culture medium. However, it is still not known if this phenomenon also occurs in other yeast strains. Therefore, the function of gene-coding gallic acid decarboxylase in the other strain need to be investigated.

In the present study, a yeast strain of $A$. melanogenum $\mathrm{T} 9$ isolated from red wine starter showed an ability for tannins degradation and its tannin tolerance was studied. Three different coding-tannase genes $\tan A$, tan $B$ and $\tan C$ and gad gene-encoded gallic acid decarboxylase, respectively, were determined and analyzed by bioinformatics method. Our result showed that $\tan A$, tan $B$ and gad gene expression level were significantly induced by tannin acid, thus the function of these genes was analyzed by gene knockout method in order to elucidate their role in tannic acid catabolism. 


\section{Materials and Methods}

\subsection{Regents and Instruments}

Tanic acid, gallic acid, pyrogallic acid, yeast extract, tryptone, glucose, $\mathrm{NH}_{4} \mathrm{NO}_{3}, \mathrm{NaCl}$, bromophenol blue, agar, and other reagents used in this study were purchased from Sangon company (Shanghai, China). Hygromycin and driselase were purchased from Solarbio company (Beijing, China). Primers were synthesized by Synbio Technologies corporation (Suzhou, China). PCR master mix and SYBR real-time PCR master mix were purchased from Thermo Scientific (Sunnyvale, CA, USA).

Microorganism cultivation was performed in the incubator and shaker of Zhichu company (Shanghai, China). qRT-PCR assay was carried out by ABI 7500 (Applied Biosystems, Arlington, VA, USA).

\subsection{Strains, Plasmids, and Media}

The strain T9 originally from red wine starter was used throughout this study and routinely grown in YPD medium containing 1.0\% yeast extract, $2.0 \%$ tryptone and $2.0 \%$ glucose. Aureobasidium melanogenum CBS 110374 was purchased from CBS-KNAW Collections. Tannins degradation test medium with $2.0 \%$ tannin, $1.0 \% \mathrm{NH}_{4} \mathrm{NO}_{3}, 0.2 \%$ bromophenol blue, and $3.0 \%$ agar was employed to determine the tannins degradation ability of the obtained yeast strains. The YPT broth medium used for analyzing the tannin degradation of strain T9 consisted of $1.0 \%$ yeast extract, $2.0 \%$ tryptone and 2.0\% tannins. Escherichia coli strains $\mathrm{DH} 5 \alpha$ were used as host strains for bacterial transformation and plasmid isolation. The strains were cultured in Luria Bertani medium supplemented with $100 \mathrm{mg} / \mathrm{L}$ ampicillin or $50 \mathrm{mg} / \mathrm{L} \mathrm{kanamycin} \mathrm{if} \mathrm{necessary.}$

\subsection{Isolation, Phylogenetic Analyses of Tannin-Degrading Yeast Strain}

Samples were collected from red wine starter and used as sources of yeast strains. The yeast isolation and screening were performed as previously reported [21]. As for the screening of tannin-utilizing yeast strains, the visual reading method was executed according to Kumar, with some modifications [22]. A single yeast colony was picked and inoculated the plate of the test medium at $28{ }^{\circ} \mathrm{C}$ for 3 days, then green to brown coloration of the medium was judged as a positive result for tannin degradation. The strain T9 showing a clear zone around the colony was used for the subsequent investigation, and its phylogenetic relationships with the typical strains reported were analyzed by amplification and sequencing of the internal transcribed spacer (ITS). A phylogenetic tree was constructed by the method of maximum likelihood to determine the phylogenetic position of the strain T9 with MEGA version 6 [23].

\subsection{Tannic Acid Tolerance Analyses by A. melanogenum T9}

The tannin degradation process of $A$. melanogenum $\mathrm{T} 9$ strain was analyzed by the estimation of cells biomass, tannase activity, the content of tannic acid, pyrogallic acid, and gallic acid. The A. melanogenum T9 yeast cells were inoculated into the YPD medium for the seed culture at $28{ }^{\circ} \mathrm{C}$, and then the seed cultures were carried out in $250 \mathrm{~mL}$ flasks containing $50 \mathrm{~mL}$ of YPT medium. The solutions of tannins of various concentrations were filter sterilized using a cellulose nitrate membrane of $25 \mathrm{~mm}$ diameter and $0.45 \mathrm{~mm}$ pore size (Whatman Ltd., Maidstone, UK). These inoculated flasks were incubated at $28^{\circ} \mathrm{C}$ with shaking and monitored at the incubation period. Biomass formed in each of the flasks was filtered through and dried at $60{ }^{\circ} \mathrm{C}$ overnight. Samples were collected every $12 \mathrm{~h}$ during the cultivation. The biomass content was gravimetrically calculated. Tannic acid was determined by the phenol-sulfuric acid method following the modifications reported by Ref. [24]. A reliable quantification of tannase activity was measured by estimating the gallic acid formed due to enzyme action [25]. One tannase unit was defined as the enzyme amount needed to release $1 \mu$ mol of gallic acid per min. Gallic acid decarboxylase was detected as Meier described [14]. Three independent assays were carried out and the average values were calculated. 


\subsection{Gene Expression Level Analyses with qRT-PCR Assay}

The seed culture of $A$. melanogenum T9 was prepared and a total of $1.0 \mathrm{~mL}$ of the seed cultures was inoculated into the YPT medium or YPD medium for $36 \mathrm{~h}$ at $28^{\circ} \mathrm{C}$. Afterwards, the yeast cells from the two samples were harvested $\left(10,000 \times g\right.$ at $\left.4{ }^{\circ} \mathrm{C}\right)$ and resuspended in $10 \mathrm{mM}$ phosphate buffer (PBS, pH 7.2-7.4). Total RNA was isolated using TRIzol reagents described by the manufacturer. RNA concentration was analyzed with NanoDrop2000c spectrophotometer (Thermo Fisher, Bremen, Germany) and reversed transcribed into cDNA. Real-time PCR was performed in triplicate using a SYBR green assay kit (Toyobo, Japan), with the SYBR real-time PCR master mix (Applied Biosystems). The primers for transcription-quantitative PCR (qRT-PCR) were designed according to the sequences of the genes. The primers were listed in Table 1. The $18 \mathrm{~S}$ rDNA gene was employed as an internal reference [26]. A complete genome sequencing of $A$. melanogenum CBS 110374 has been accomplished and was then selected as reference data for analyses. Three putative genes $\tan A, \tan B$ and $\tan C$ correspondingly coding tannase of TanAp (NCBI access number MN295986), TanBp (NCBI access number MN295987) and TanCp (NCBI access number MN295988) respectively, and gene gad coding gallic acid decarboxylase (GAD, NCBI access number MN295989), were found and annotated in Aureobasidium melanogenum T9. Relative gene expression level changes were analyzed by using the comparative CT method with a $10-\mu \mathrm{L}$ reaction system. Samples from YPD medium were used as control. All of the amplifications were performed in triplicate from biological triplicates.

Table 1. The primers used for qRT-PCR analyses in this study.

\begin{tabular}{cc}
\hline Primers & Sequence $\mathbf{5}^{\prime} \mathbf{- 3}^{\prime}$ \\
\hline TA5 & CGATTGGAGCACCTTCAACGAGA \\
TA3 & CGACCTGAAAGGGATGATGGGAT \\
TB5 & TATTCGTGTTGTAGGTCGGGTCA \\
TB3 & AGAACGGCACCATTACTGCTCAA \\
TC5 & GACCTCGACGTAACCAGACCTGA \\
TC3 & CAACTGACGATGTTCCTTGCTCC \\
GD5 & CAACAAGTTGGAAGCAAGGCAATA \\
GD3 & CACAGCACGAGTGAGGTTGGGAT \\
\hline
\end{tabular}

2.6. Proteins Expression Level Analyses with Label-Free Technology Mass Spectrometry-Based Label-Free Quantitative Proteomics

Proteins expression-level quantitative analyses of TanAp, TanBp, TanCp and GAD were measured by mass spectrometry-based label-free quantitative proteomic technology. For this purpose, $2 \mathrm{~mL}$ cultures from the YPT medium or YPD medium were centrifuged for $10 \mathrm{~min}$ at $4{ }^{\circ} \mathrm{C}$ with $10,000 \times g$. Five-hundred microliters of supernatant was adjusted to $\mathrm{pH} 2$ with $\mathrm{HCl}$, and compounds were extracted with $1 \mathrm{~mL}$ MTBS (Sigma-Aldrich, USA). The obtained total proteins of yeast cells were then digested and identified by liquid chromatography (LC)-electron spray ionization (ESI)-tandem mass spectrometry (MS/MS) analyses. For proteins quantitation, these proteins were weighted and normalized relative to the median ratio in NovoGene Corporation (Beijing, China).

\subsection{Genes Function Analyses by Construction of Mutant Strains}

Gene disruptions were performed as described by Chi [27]. According to the sequence of the disruption vector, a fragment containing Poly(A)-hygromycin B phosphotransferase (HPT) gene-TEF promoter was synthesized (Synbio Technologies, China). Primers A5F, A5R, A3F, and A3R were designed according to the coding sequence (CDS) of $\tan A$. Primers A5F and A5R were used to amplify the $5^{\prime}$-arm; Primers A3F and A3R were used to amplify the $3^{\prime}$-arm. Primers HPT5 and HPT3 were used to amplify the fragment Poly(A)-hygromycin B phosphotransferase (HPT) gene-TEF promoter. Then, the $5^{\prime}$-arm, $3^{\prime}$-arm, and DNA fragment containing Poly(A)-hygromycin B phosphotransferase (HPT) gene-TEF promoter were connected by overlapping PCR, generating the $\tan A-$ knocking fragment. 
This obtained fragment was transformed into the competent cells. The disruptants were grown on the YPD plate containing $50.0 \mu \mathrm{g} / \mathrm{mL}$ hygromycin for 2 days. One colony was picked as strain $\Delta \tan A$. Primers B5F and B5R were used to amplify the $5^{\prime}$-arm of the $\tan B$-knocking fragment; primers B3F and B3R were used to amplify the $3^{\prime}$-arm of the tanB-knocking fragment. Primers G5F and G5R were used to amplify the $5^{\prime}$-arm of the gad-knocking fragment; primers G3F and G3R were used to amplify the $3^{\prime}$-arm of the gad-knocking fragment. $\tan B$-knocking fragment and gad-knocking fragment were constructed as above; the genes of $\tan B$ and $g a d$ were disrupted using the same method. To check the synergistic effect of $\tan A$ and $\tan B$, strain $\Delta \tan A$, strain $\Delta \tan B$, strain $\Delta g a d$, and wild strain T9 were prepared, and a total of $1.0 \mathrm{~mL}$ of the seed cultures was inoculated into the YPT medium for $36 \mathrm{~h}$ at $28^{\circ} \mathrm{C}$. Gene expression level analyses of $\tan A$ and $\tan B$ were carried out with qRT-PCR assay as described earlier in the paper. The primers used for the construction of mutant strains are displayed in Table 2.

Table 2. The primers used in this study. The underlined boxed bases are the shared bases.

\begin{tabular}{cc}
\hline Primers & ${\text { Sequence } \text { 5' }^{\prime} \mathbf{3}^{\prime}}^{\text {A5F }}$ \\
A5FTGGAACTCGTCCTACAACAT \\
A5R & GATCCCCCGAATTAGACCTGCATCTCCTTCAGTCCTT \\
A3F & ATGAGCCAACTGTCGCCGAGCCCTACGATTGGAGCACCTT \\
A3R & GGTTGAGTAGCGCCAGCGATGTA \\
B5F & GTCGATGGAAGCCTTGTCGTGTA \\
B5R & GATCCCCCGAATTACACTTATCCTGACCTGACCACCTT \\
B3F & ATGAGCCAACTGTCGAAAGGGAGAAACCACCTGGCAATT \\
B3R & TCCAACCAGCCATGAGTCACCTC \\
G5F & ATGAAGGTTCGCGAGATCTGTGAGG \\
G5R & GATCCCCCGAATTAATGTCGACTTGGGAGCCGATGATGC \\
G3F & ATGAGCCAACTGTCGAGATGGACAATGACGCCGACTGTCG \\
G3R & GATCATCCTCACCAGTCAAATCAGG \\
HPT5 & TAATTCGGGGGATCTGGATTTTAGTACTGGA \\
HPT3 & CGACAGTTGGCTCATCATCCGTTACATCA
\end{tabular}

\subsection{GAD and Different Tananses Proteins Analyses with Bioinformatics Method}

The bioinformatics analyses of TanAp, TanBp, TanCp, and GAD were conducted. Database searches were obtained from NCBI website (https://www.ncbi.nlm.nih.gov/) and performed using BlastX (http://www.ncbi.nlm.nih.gov/BLAST), and multiple sequence alignments were analyzed with DNAman software package (Version 5.2.2, Lynnon Biosoft, Quebec, Canada). Protein analyses with secretion signal peptide prediction was conducted according to the SignalP (version 4.1) program (http://www.cbs.dtu.dk/services/SignalP/). Physiochemical data were generated from the ProtParam software using ExPASy server (the proteomic server of Swiss Institute of Bioinformatics). A phylogenetic tree of TanAp, TanBp and TanCp as well as other putative tannase protein sequences from fungi and yeast were developed using the Neighbor-Joining method.

\subsection{Statistical Analyses}

The data were the average of three independent experiments, and the error bars indicated the standard deviations (SDs) from the mean of triplicates. The significant differences among groups were calculated with analyses of variance (ANOVAs) followed by Dunnett's tests by using GraphPad Prism 5 software (San Diego, CA, USA). $p$ values of 0.05 were defined as statistically significant in our study.

\section{Result}

\subsection{A. melanogenum T9 Having the Ability of Tannic Acid Degradation}

After isolation and purification of the yeast strains from the red wine starter samples, it was found that the morphologies of 20 obtained yeast strains were similar to those of Aureobasidium spp. The 20 
strains were cultivated in tannins degradation test medium and evaluated by their abilities to degrade tannins. Nine strains grew in the medium, indicating that these strains have the ability of tannic acid degradation. Compared with other strains, T9 has great potential for tannins degradation (Figure S1). Also, there was an obvious change from blue to yellow in the test medium (data not shown), which indicated that tannic acid was transformed into a more acidic substance. The colonies of the strain T9 grown on the potato dextrose solid medium had yeast-like cells and the colonies are observed as cream, exhibiting characteristics of Aureobasidium spp. Furthermore, ITS sequencing and phylogenetic tree construction of the strain T9 were performed as described in "Materials and methods". According to the high homology (99\% identity) with A. pullulans var. melanogenum CBS109800, confirmed by topology of the phylograms, the strain T9 was assigned to A. melanogenum T9 (data not shown). The genome of the type of strain of this species, A. melanogenum CBS 110374, has been sequenced, providing convenience for our study. A. melanogenum CBS 110374 also has the ability for tannins degradation, but obviously weaker than strain T9 (Figure S1).

\subsection{Analyses of Tannin Tolerance by A. melanogenum T9}

For the tannin tolerance assay, the A. melanogenum T9 strains were cultivated in the YPT medium with different concentrations of tannin $(10,15,20,25$, and $30 \mathrm{~g} / \mathrm{L})$. Figure 1 a showed that the biomass of A. melanogenum T9 increased with the tannin's concentration rising and peaked off early with $6.7 \pm 0.3 \mathrm{~g} / \mathrm{L}$ under the condition of $20 \mathrm{~g} / \mathrm{L}$. Nevertheless, the growth inhibition was observed when the tannin's content was beyond $20 \mathrm{~g} / \mathrm{L}$, implying that the excess tannins had adverse effects for A. melanogenum T9 growth. Furthermore, with the increase of tannin concentration in the medium, the gallic acid content had a clear rising trend, showing that tannic acid was degraded into gallic acid by A. melanogenum T9.
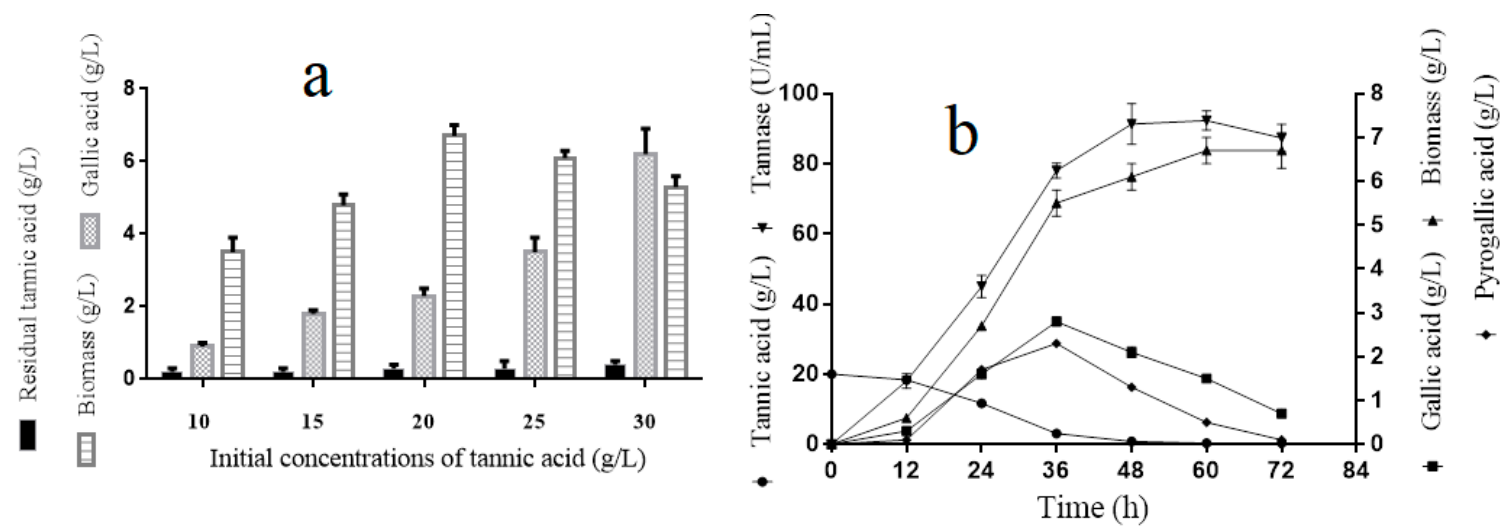

Figure 1. Tannic acid tolerance analyses of A. melanogenum T9 under the conditions of different tannin acid concentrations (a). Tannic acid degradation process analyses for A. melanogenum T9 (b). The mean expression values \pm SDs are reported relative to the control.

\subsection{A. melanogenum T9 Growth Process Analyses with Tannic Acid as the Sole Carbon Course}

The results in Figure $1 \mathrm{~b}$ showed that the biomass and tannase activity of $A$. melanogenum T9 reached a relative stable level at $48 \mathrm{~h}$. Tannins had an obvious content decline and were totally decomposed after 40-h culture. Meanwhile, with the decrease of tannic acid content, the yield of gallic and pyrogallic acid increased obviously and reached the highest values at $36 \mathrm{~h}$, with $3.1 \pm 0.1 \mathrm{~g} / \mathrm{L}$ and $2.8 \pm 0.1 \mathrm{~g} / \mathrm{L}$, respectively.

\subsection{Bioinformatics Analyses of Tannases and GAD}

In the A. melanogenum T9, tannase of TanAp, TanBp and TanCp contained 528, 587 and 508 amino acids respectively, with predicted protein molecular masses of 57.2, 63.4 and 54.7 kDa accordingly. 
Proteins belonging to three different tannases shared $18.14 \%$ amino acids identity. The multiple sequence alignment of these three tannase protein sequences showed several putative conserved domains, pentapeptide motif Gly-X-Ser-X-Gly typical of serine hydrolases and (acetyl esterase/lipase) and Abhydrolase_3 (alpha/beta hydro-lase fold). The phylogenic tree showed that although the three different tannases had common roots, they diverged in three branches within which they evolved along discrete lines to form subclasses of specialized enzymes (Figure 2a). Therefore, it seemed that TanAp, TanBp and TanCp could be classified into three different tannase enzymes and these proteins' biochemical characteristics are predicted in Table 3. The data showed that the signal peptide appeared in three different tannases and their evolutionary relationship closed proteins. Though the molecule weight can be used to distinguish TanAp-like tannases (molecule weight from 55.15 to $57.23 \mathrm{kDa}$ ) and TanBp-like tannases (molecule weight from 62.57 to $65.65 \mathrm{kDa}$ ), it was not suitable to differentiate TanCp-like (molecule weight from 43.54 to $81.47 \mathrm{kDa}$ ) from other types of tannases.

The putative GAD protein had an open reading frame of $696 \mathrm{bp}$, encoding a protein with 232 amino acids. The predicted molecular mass of GAD is $27.3 \mathrm{kDa}$, and protein analyses revealed the absence of a secretion signal sequence. The phylogenetic tree (Figure 2b) inferred from 1000 bootstrap replicates is taken to represent the evolutionary relationship between GAD and other putative gallic acid decarboxylase protein sequences from yeast and fungi. These proteins, including hypothetical protein (THV69751.1), hypothetical protein (KEQ79373.1), hypothetical protein (THV90437.1), and gallate decarboxylase (SJN60119.1), had a 71.56\% similarity of protein sequences. The proposed putative enzymes of $A$. pullulans originated from the same ancestral node, which shares a common ancestor with GAD. The conclusion is that GAD has a closely-related evolutionary relationship with gallic acid decarboxylases from A. pullulans and Blastobotrys adeninivorans.

Table 3. The property analysis of TanAp-, TanBp-, and TanCp-like tannases.

\begin{tabular}{|c|c|c|c|c|c|}
\hline Protein & Accession Number & Strain & Residues (aa) & MW (KDa) & Signal Peptide \\
\hline \multicolumn{6}{|c|}{ TanAp-Like Proteins } \\
\hline Tannase & KEQ59156.1 & A. melanogenum CBS 110374 & 528 & 57.23 & Yes \\
\hline Tannase & KEQ86878.1 & Aureobasidium pullulans EXF-150 & 542 & 55.15 & No \\
\hline Tannase & OAK97256.1 & Stagonospora sp. SRC1lsM3a & 538 & 56.57 & No \\
\hline Tannase & EJD50919.1 & Auricularia subglabra TFB-10046 SS5 & 537 & 53.72 & No \\
\hline Tannase & KEQ62357.1 & A. melanogenum CBS 110374 & 587 & 63.39 & No \\
\hline Tannase & KEQ81812.1 & A. pullulans EXF-150 & 583 & 62.95 & No \\
\hline Tannase & EYE96818.1 & Aspergillus ruber CBS 135680 & 579 & 62.76 & No \\
\hline Tannase & KXG49722.1 & Penicillium griseofulvum & 580 & 62.85 & Yes \\
\hline Tannase & PQE10574.1 & Rutstroemia sp. NJR-2017a BVV2 & 581 & 62.62 & Yes \\
\hline Tannase & CRL25663.1 & Penicillium camemberti & 580 & 62.86 & Yes \\
\hline Tannase & OKP13191.1 & Penicillium subrubescens & 589 & 64.00 & No \\
\hline \multicolumn{6}{|c|}{ TanCp-like proteins } \\
\hline Tannase & KEQ62631.1 & A. melanogenum CBS 110374 & 508 & 54.68 & Yes \\
\hline Tannase & KEQ81081.1 & A. pullulans EXF-150 & 496 & 53.94 & No \\
\hline Tannase & XP_020125410.1 & Diplodia corticola & 542 & 58.16 & No \\
\hline Tannase & PVH72277.1 & Cadophora sp. DSE1049 & 513 & 54.75 & Yes \\
\hline Tannase & XP_018037725.1 & Paraphaeosphaeria sporulosa & 508 & 55.17 & Yes \\
\hline Tannase & КХН32567.1 & Colletotrichum nymphaeae SA-01 & 468 & 51.07 & No \\
\hline Tannase & XP_018070318.1 & Phialocephala scopiformis & 405 & 43.54 & No \\
\hline
\end{tabular}



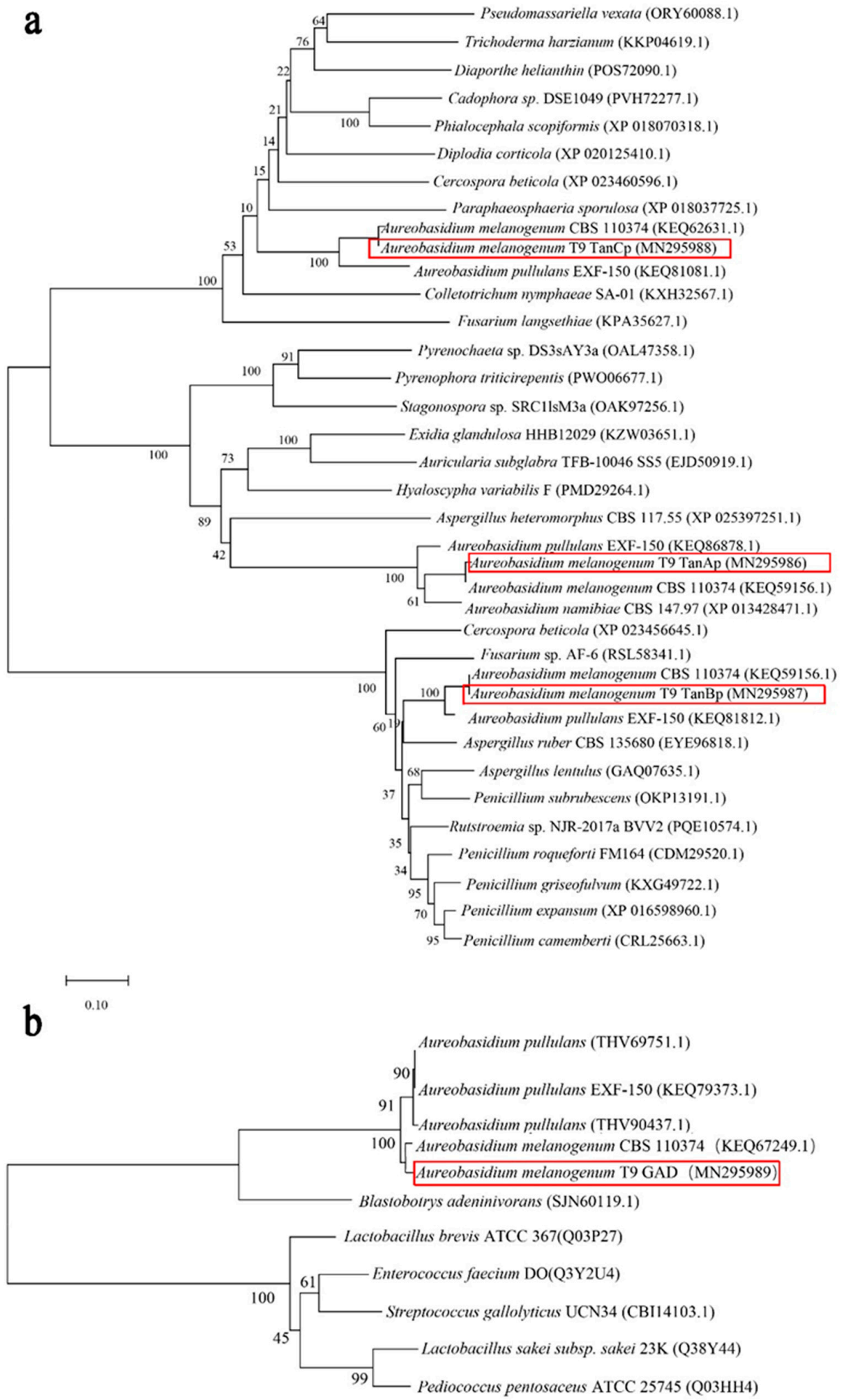

0.20

Figure 2. The phylogenetic tree of TanAp, TanBp and TanCp, and tannases from other yeasts and fungal species (a); the phylogenetic tree of GAD and gallic acid decarboxylases from other yeasts and fungal species (b). 


\subsection{Tannic Acid Induced Related Genes Expression Up-Regulation}

In Table 4, qRT-PCR data showed that the gene relative expressions of $\tan A$ and $\tan B$ cultured in YPT medium were 32-fold and 64.70-fold increased respectively compared to those cultured in YPD medium. Furthermore, the data of Mass Spectrometry-Based Label-Free Quantitative Proteomics demonstrated that these proteins' expression levels of TanAp and TanBp were 8.22-fold and 332.00-fold higher than those of the control. However, no obvious transcriptional level difference of the $\tan C$ was observed when the A. melanogenum T9 was exposed to tannins. Besides, gad also showed a 3.21-fold up-regulation change as compared to that of the control.

Table 4. The transcription level and translation level changes of the genes cultured in YPT medium compared to those cultured in YPD medium.

\begin{tabular}{cccc}
\hline Gene & Protein & Gene Transcription Level Change (Fold) & Protein Translation Level Change (Fold) \\
\hline $\tan A$ & TanAp & $32.00 \pm 3.6$ & 8.22 \\
$\tan B$ & TanBp & $64.70 \pm 5.2$ & 332.00 \\
$\tan C$ & TanCp & $0.74 \pm 0.2$ & - \\
gad & GAD & $3.21 \pm 0.3$ & - \\
\hline
\end{tabular}

\section{6. $\tan A$ and $\operatorname{tanB}$ Having Sililar Function on the Tannic Acid Metabolizing}

The above-mentioned assay results showed that genes $\tan A, \tan B$ and gad could be induced by tannic acid. Thus, their role in tannin acid degradation were further analyzed. As shown in Figure 3e, the tannase activity of $A$. melanogenum T9 achieved stability at $48 \mathrm{~h}$ with $91.3 \pm 5.8 \mathrm{U} / \mathrm{mL}$, which was much higher than that of $\triangle \tan A$ and $\triangle \tan B$ with $51.3 \pm 4.1$ and $64.1 \pm 1.9 \mathrm{U} / \mathrm{mL}$, respectively. On account of tannic acid being used as the sole carbon course in the medium, the tannic acid utilization ratio would have a direct impact on the growth and reproduction of yeast cells. Thus, the tannin degradation and biomass accumulation rate declined, especially within the first $30 \mathrm{~h}$ of culture (Figure $3 \mathrm{a}, \mathrm{b}$ ), which was exclusively due to tannase activity reduction in the mutants $\triangle \tan A$ and $\triangle \tan B$. Even so, mutants $\triangle \tan A, \triangle \tan B$ and wild-type degraded all of tannic acid at $72 \mathrm{~h}$, and their biomass finally reached the same level at $60 \mathrm{~h}$.
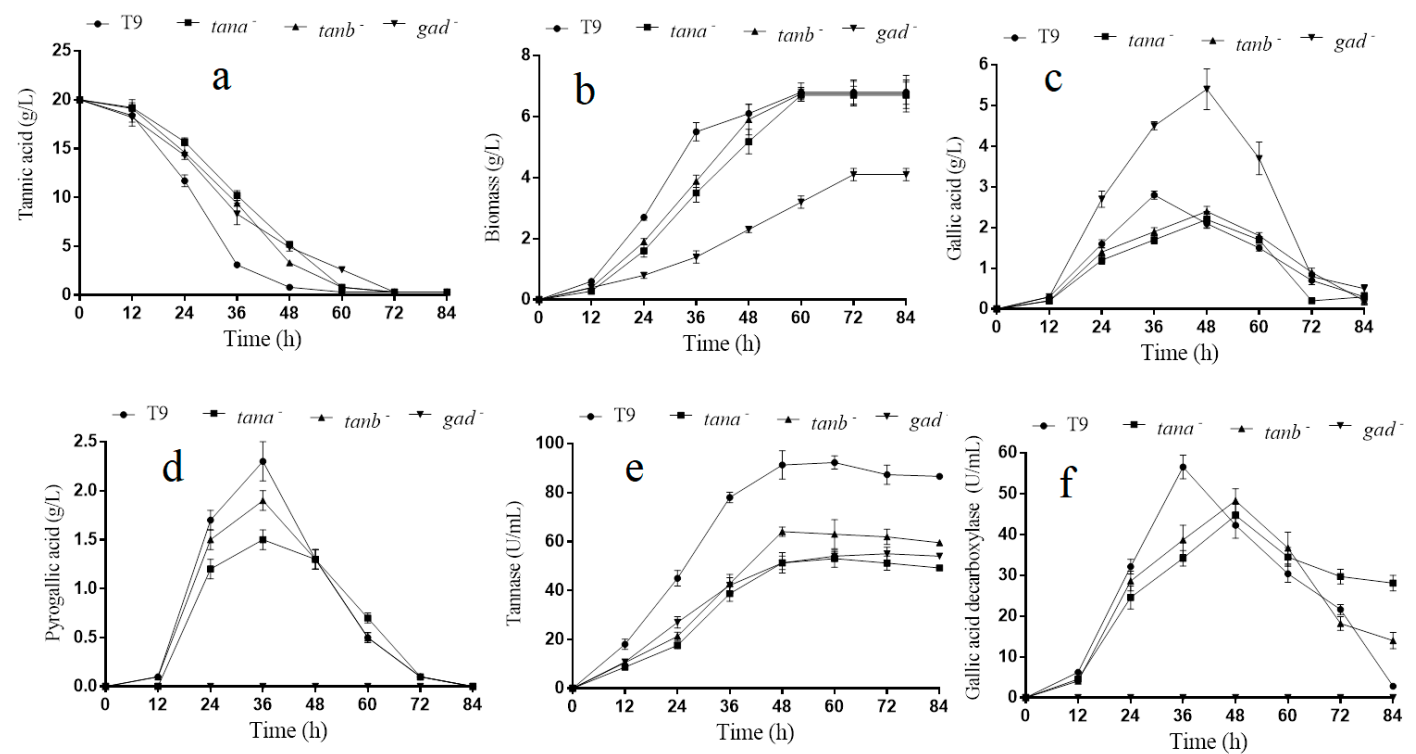

Figure 3. Tannic acid degradation by mutants and A. melanogenum T9 (a); biomass change of mutants and A. melanogenum T9 (b); gallic acid content change of mutants and A. melanogenum T9 (c); pyrogallic acid content change of mutants and $A$. melanogenum $\mathrm{T} 9$ (d); tannase activity change of mutants and A. melanogenum T9 (e); gallic acid decarboxylase activity change of mutants and A. melanogenum T9 (f). Data are given as means $\pm \mathrm{SD}, n=3$. 
Furthermore, the concentration analyses of gallic acid and pyrogallic acid were performed on the yeast cells of the $\Delta \tan A, \Delta \tan B$ and wild-type grown for $84 \mathrm{~h}$ (Figure 3c,d). It could be seen that the appearance time of maximum concentration of gallic acid in both strains $\Delta \tan A$ and $\Delta \tan B$ was $48 \mathrm{~h}$, which was significantly delayed when compared to wild-type with $36 \mathrm{~h}$. Meanwhile, the gallic acid maximum concentration of $\Delta \tan A$ and $\Delta \tan B$ was also lower than the wild-type strain. The appearance time delay and decline of maximum gallic acid concentration in $\triangle \tan A$ and $\Delta \tan B$ may partly be attributed to the reduction of tannase activity and tannic acid degradation rate. Moreover, the relative transcription level of gene $\tan B$ in $\triangle \tan A$ increased to $183.2 \%$, compared with that in the wild strain; the relative transcription level of gene $\tan A$ in $\triangle \tan B$ increased to $121.2 \%$, compared with that in the wild strain (Figure S2). In the $\triangle g a d$ strain, the relative transcription level of both genes of $\tan A$ and $\tan B$ decreased significantly (Figure S2).

\subsection{Gad Was Crucial for on the Tannic Acid Metabolizing and Growth}

In Figure $3 d, f$, our experiment result showed that no release of pyrogallic acid and gallic acid decarboxylase activity was seen during the entire cultivation process in the mutant $\Delta g a d$ compared to the parental strain. As gallic acid decarboxylase could catalyze gallic acid into pyrogallic acid, it can be easily concluded that there was only one gene encoding decarboxylase in the A. melanogenum T9. The decarboxylase activity disappearing further resulted in the excessive accumulation of gallic acid within the first $60 \mathrm{~h}$ of incubation. However, the accumulative gallic acid was finally almost completely degraded by $\Delta$ gad, suggesting that gallic acid was utilized by $A$. melanogenum T9 by other unknown metabolic pathways (Figure 3c). The quantitative analyses data showed that an overall $39.7 \%$ reduction in the biomass was found for the mutant $\Delta g a d$ compared with A. melanogenum T9 after a 72-h culture was conducted (Figure 3b). Additionally, it was worth noting that the gene AGDC1 deletion also led to the reduction of tannase activity and tannic acid degradation rate (Figure 3a,e).

\section{Discussion}

Tannins are generally considered recalcitrant to biodegradation and have toxic effects on various organisms. However, some microorganisms have evolved to use tannins as carbon and energy sources. Up to now, most of the microorganism species used for tannin biodegradation were determined as fungi or bacteria, few researches were related with yeast. In this study, a tannin-degrading yeast strain of Aureobasidium melanogenum T9 was isolated and identified from the red wine starter. A. melanogenum T9 was able to grow well under conditions of different contents of tannin and it had the highest biomass when an initial tannic acid concentration of $20 \mathrm{~g} / \mathrm{L}$ was used (Figure $1 \mathrm{~b}$ ). Bi Shi reported that tannin tolerances of $C$. utilis was adapted to $25 \mathrm{~g} \mathrm{dm}^{-3}$ by gradually increasing the concentrations of valonea tannins in the culture media, and the most suitable concentration of valonea tannins in liquid fermentation medium for ellagic acid production by C. utilis was $9.0 \mathrm{~g} \mathrm{dm}^{-3}$ [15].

Multiple sequence alignment of TanAp, TanBp and TanCp protein sequences displayed the putative conserved domains of Gly-X-Ser-X-Gly and Abhydrolase_3. The conserved signature sequence "Gly-X-Ser-X-Gly" of motif was unprecedented in serine hydrolases and showed the highest conservancy among all of kinds of bacteria and fungi tannase sequences [28]. The mutational analyses indicated that the disulfide bond between Cys202 and Cys458 is crucial for the activity of AoFaeB [29]. The different types of tannase proteins found in the same yeast or fungal species may result from the cumulative effect of species specificity and varying interaction with external environments. In bacteria, the absence signal peptide as well as molecular size were used to help to identify different subtype tannases; nevertheless, these characteristics were improved for tannases of yeast and fungi. Additionally, the phylogenetic relation between GAD and other similar proteins were identified, and these proteins had a high similarity. In the bacteria, like Lactobacillus plantarum, several conserved domains, such as subunit B, were fundamental for enzyme catalytic activity and structure in the Agdc1p of Lactobacillus plantarum WCFS1 [30]. 
Tannase can be induced by phenolic compounds such as gallic acid, pyrogallol, methyl gallate, and tannic acid. However, the induction mechanism has not been demonstrated on the gene expression level [31]. Similar to those of previous researches, our assay result showed that the tannase activity had remarkable rise when tannin was present in the medium. The qRT-PCR assays exhibited the inducibility of gene $\tan A$ and $\tan B$, nevertheless, no significant difference of $\tan C$ was noticed at the level of gene transcription level in the presence of tannins. It is interesting that the $\tan C$ gene was not sensitive to the tannins, and whether $\tan C$ was involved in tannins degradation needs to be explored. These results demonstrated that although the tannase synthesis was induced at the protein expression level by tannin, not every tan gene coding tannase was induced by tannin at the gene transcription level. In addition, our assay result showed that tannin also caused the gad gene expression level to up-regulate in $A$. melanogenum. Thus, the $\tan A, \tan B$ and $\mathrm{gad}$ gene functions in the tannin degradation process were explored in the subsequent assays.

As expected, the tannase activity had a remarkable decline after gene $\tan A$ or $\tan B$ was knocked-out (Figure 3e). The reduction of tannase activity further caused tannin degradation, biomass accumulation rate decreased as well as the appearance time delay, and gallic acid maximum concentration declined (Figure $3 \mathrm{a}-\mathrm{c}$ ). However, mutants $\triangle \tan A, \Delta \tan B$, and the origin strain did not exhibit biomass differences, and tannins were nearly completely utilized by these strains after an 84-h culture. Of note, $\triangle \tan A$ and $\triangle \tan B$ had a rather high similarity in the biomass accumulative and enzyme activity change trend. It could be concluded that the gene $\tan A$ performed a similar function in tannin degradation with $\tan B$. However, the existence of single $\tan A$ or $\tan B$ was enough for the strain to utilize tannins, although causing a growth delay to some extent. No obvious synergistic effect of the two enzymes was observed. These two enzymes may be responsible for catalyzing the same reactions. To further study this, the two enzymes should be expressed and characterized.

The mutation of any of those two genes induces a higher transcription of the other (Figure S2). Double-gene disruption of $\tan A$ and $\tan B$ will be carried out in future work, which may cause more significant defects in tannin degradation. The three tannase genes in this study were found based on the sequence similarity and were confirmed by the RNA-seq data and label-free quantitative proteomics. However, in the RNA-seq data, some genes annotated with unknown functions have been found with a certain degree of structural similarity with the identified tannases. In future work, the functions of these genes should be identified.

Additionally, the role of gene gad on the tannin degradation was also explored. The quantitative determination of pyrogallic acid content and gallic acid decarboxylase activity from $\Delta g a d$ and wild-type (Figure 3d,f) demonstrated that gad was the only gene responsible for the gallic acid decarboxylase synthesis in the A. melanogenum T9 under this culture condition. Although the gad disruption prevented gallic acid effective degradation in some way, the excessive gallic acid was still entirely decomposed by the $A$. melanogenum T9 with other unknown pathways. Usually, gallic acid decarboxylase plays a very important role in the metabolism of tannins and phenol derivatives. However, a recent research exhibited that the deletion of gene AGDC1 from A. adeninivorans also contributed to cells death or growth inhibition when gallic acid was present in culture medium under this culture condition [14]. Similar to a previous report, a growth inhibition phenomenon was noticed in the mutant $\Delta g a d$ utilizing the tannic acid as the sole carbon source; how the gene gad imposed an adverse impact on the yeast growth remains unclear. It is possible that the excessive gallic acid caused by a lack of gallic acid decarboxylase had a toxic effect on $A$. melanogenum $\mathrm{T} 9$, or the gene gad played a key role in the yeast cells growth. Thus, the retailed mechanism needs to be explored in the future. In A. melanogenum T9, the gene gad deletion has been associated with reduction of the tannase activity and tannic acid degradation rate during the tannic acid degradation process. It is possible that the tannase activity decline in the mutant $\Delta g a d$ may have resulted from the excessive accumulation of gallic acid. A high concentration of gallic acid may have negative feedback regulation on tannase activity, and tannase activity decline was associated with a concomitant decrease in the tannic acid degradation rate. 
Biodegradation by certain microorganisms and enzymes is one of the most efficient ways to degrade large tannin molecules into small molecules with bio-activities of high value, such as gallic acid or pyrogallol [32]. Nowadays, tannase and other metabolites, like gall acid and pyrogallol, secreted by tannin-degrading microorganisms, are gaining more attention because of their industrial importance. Tannase has a long study history over more than a century and an extensive biotechnological application in the field of food stuff detannification and chemical and healthcare product manufacturing [33]. Gallic acid has been used in food, cosmetics, adhesives, manufacture of writing inks and dyes, and the synthesis of trimethoprim in the pharmaceutical industry $[34,35]$. As the major hydrolytic product of gallic acid, pyrogallic acid was used for leathers staining, hair coloring, photographic plate-developing agent, and anti-tumor drug [19]. On the other hand, the yeast $A$. melanogenum is often used as a host for producing high levels of extracellular proteins and products, since it can grow in simple defined media and reaches very high cell densities. In addition, several studies have shown that the direct use of these strains and their metabolites are safe in the human-related sectors, such as in the medical and food industries [3]. Thus, A. melanogenum could be an important candidate for future use in tannin-related industries.

\section{Conclusions}

In this study, several genes crucial for tannin degradation in Aureobasidium melanogenum were revealed for the first time by integrated approaches. The optimal tannic acid concentration for A. melanogenum T9 growth was supposed to be $20 \mathrm{~g} / \mathrm{L}$. The genes encoding tannases and gallic acid decarboxylase were analyzed respectively by gene knock-out method in order to better understand their role during the tannin degradation process. The result revealed that mutants $\triangle \tan A$ and $\triangle \tan B$ had a much lower tannase activity and tannin acid degradation rate than that of the parental strain. Furthermore, no gallic acid decarboxylase activity and pyrogallic acid were detected after gene gad was deleted. The gene gad knock-out not only led to the drop of tannase activity and the tannic acid degradation rate, but also exerted a negative impact on the growth of A. melanogenum T9. The above experimental results provided new insights for the mechanism of tannins biodegradation by yeast and also showed that $A$. melanogenum has the potential for the production of gallic acid and pyrogallol.

Supplementary Materials: The following are available online at http://www.mdpi.com/2218-273X/9/9/439/s1, Figure S1: Tannic acid degradation by different Aureobasidium spp. strains, Figure S2: Transcriptional level analysis of $\tan A$ and $\tan B$ genes in different strains.

Author Contributions: Conceptualization, Z.-P.W.; Data curation, S.L. and J.L.; Investigation, L.-L.Z.; Methodology, X.-J.Y.; Resources, S.L.; Software, Y.-L.W. and S.L.; Supervision, Z.-P.W.; Writing—original draft, L.-L.Z. and Y.-L.W.; Writing-review \& editing, J.L. and Z.-P.W.

Funding: This research was supported by the National Natural Science Foundation of China [grant number 31500032].

Conflicts of Interest: The authors declare that they have no competing interests.

\section{References}

1. Chi, Z.; Wang, F.; Chi, Z.; Yue, L.; Liu, G.; Zhang, T. Bioproducts from Aureobasidium pullulans, a biotechnologically important yeast. Appl. Microbiol. Biotechnol. 2009, 82, 793-804. [CrossRef] [PubMed]

2. Bozoudi, D.; Tsaltas, D. The Multiple and Versatile Roles of Aureobasidium pullulans in the Vitivinicultural Sector. Fermentation 2018, 4, 85. [CrossRef]

3. Prasongsuk, S.; Lotrakul, P.; Ali, I.; Bankeeree, W.; Punnapayak, H. The current status of Aureobasidium pullulans in biotechnology. Folia Microbiol. 2018, 63, 129-140. [CrossRef] [PubMed]

4. Zou, X.; Zhou, Y.; Yang, S.T. Production of polymalic acid and malic acid by Aureobasidium pullulans fermentation and acid hydrolysis. Biotechnol. Bioeng. 2013, 110, 2105-2113. [CrossRef] [PubMed]

5. An, C.; Ma, S.J.; Chang, F.; Xue, W.J. Efficient production of pullulan by Aureobasidium pullulans grown on mixtures of potato starch hydrolysate and sucrose. Braz. J. Microbiol. 2017, 48, 180-185. [CrossRef] [PubMed] 
6. Mulay, Y.R.; Deopurkar, R.L. Purification, characterization of amylase from indigenously isolated aureobasidium pullulans Cau 19 and its bioconjugates with gold nanoparticles. Appl. Biochem. Biotechnol. 2018, 184, 644-658. [CrossRef] [PubMed]

7. Liu, Y.Y.; Chi, Z.; Wang, Z.P.; Liu, G.L.; Chi, Z.M. Heavy oils, principally long-chain n-alkanes secreted by Aureobasidium pullulans var. melanogenum strain P5 isolated from mangrove system. J. Ind. Microbiol. Biotechnol. 2014, 41, 1329-1337. [PubMed]

8. Singh, R.S.; Kaur, N. Understanding response surface optimization of medium composition for pullulan production from de-oiled rice bran by Aureobasidium pullulans. In Food Science Biotechnology; Springer: Singapore, 2019; pp. 1-14.

9. Chávez-González, M.; Rodríguez-Durán, L.V.; Balagurusamy, N.; Prado-Barragán, A.; Rodríguez, R.; Contreras, J.C.; Aguilar, C.N. Biotechnological advances and challenges of tannase: An overview. Food Bioprocess Technol. 2012, 5, 445-459. [CrossRef]

10. Makkar, H.P.S.; Singh, B.; Kamra, D.N. Biodegradation of tannins in oak (Quercus incana) leaves by Sporotrichum pulverulentum. Lett. Appl. Microbiol. 1994, 18, 39-41. [CrossRef]

11. Kumar, R.A.; Gunasekaran, P.; Lakshmanan, M. Biodegradation of tannic acid by Citrobacter freundii isolated from a tannery effluent. J. Basic Microbiol. Int. J. Biochem. Physiol. Genet. Morphol. Ecol. Microorg. 1999, 39, 161-168.

12. Belmares, R.; Contreras-Esquivel, J.C.; Rodríguez-Herrera, R.; Coronel, A.R.; Aguilar, C.N. Microbial production of tannase: An enzyme with potential use in food industry. LWT Food Sci. Technol. 2004, 37, 857-864. [CrossRef]

13. Mingshu, L.; Kai, Y.; Qiang, H.; Dongying, J. Biodegradation of gallotannins and ellagitannins. J. Basic Microbiol. 2006, 46, 68-84. [CrossRef] [PubMed]

14. Meier, A.K.; Worch, S.; Böer, E.; Hartmann, A.; Mascher, M.; Marzec, M.; Bode, R. Agdc1p-a Gallic Acid Decarboxylase Involved in the Degradation of Tannic Acid in the Yeast Blastobotrys (Arxula) adeninivorans. Front. Microbiol. 2017, 8, 1777. [CrossRef] [PubMed]

15. Shi, B.; He, Q.; Yao, K.; Huang, W.; Li, Q. Production of ellagic acid from degradation of valonea tannins by Aspergillus niger and Candida utilis. J. Chem. Technol. Biotechnol. Int. Res. Process Environ. Clean Technol. 2005, 80, 1154-1159.

16. De las Rivas, B.; Rodríguez, H.; Anguita, J.; Muñoz, R. Bacterial tannases: Classification and biochemical properties. Appl. Microbiol. Biotechnol. 2019, 103, 603-623. [CrossRef] [PubMed]

17. Aguilar, C.N.; Rodríguez, R.; Gutiérrez-Sánchez, G.; Augur, C.; Favela-Torres, E.; Prado-Barragan, L.A.; Contreras-Esquivel, J.C. Microbial tannases: Advances and perspectives. Appl. Microbiol. Biotechnol. 2007, 76, 47-59. [CrossRef]

18. Gostinčar, C.; Ohm, R.A.; Kogej, T.; Sonjak, S.; Turk, M.; Zajc, J.; Sharma, A. Genome sequencing of four Aureobasidium pullulans varieties: Biotechnological potential, stress tolerance and description of new species. BMC Genom. 2014, 15, 549. [CrossRef]

19. Zeida, M.; Wieser, M.; Yoshida, T.; Sugio, T.; Nagasawa, T. Purification and characterization of gallic acid decarboxylase from Pantoea agglomerans T71. Appl. Environ. Microbiol. 1998, 64, 4743-4747.

20. Jana, A.; Halder, S.K.; Banerjee, A.; Paul, T.; Pati, B.R.; Mondal, K.C.; Mohapatra, P.K.D. Biosynthesis, structural architecture and biotechnological potential of bacterial tannase: A molecular advancement. Bioresour. Technol. 2014, 157, 327-340. [CrossRef]

21. Ma, Z.C.; Fu, W.J.; Liu, G.L.; Wang, Z.P.; Chi, Z.M. High-level pullulan production by Aureobasidium pullulans var. melanogenium P16 isolated from mangrove system. Appl. Microbiol. Biotechnol. 2014, 98, 4865-4873. [CrossRef]

22. Kumar, M.; Rana, S.; Beniwal, V.; Salar, R.K. Optimization of tannase production by a novel Klebsiella pneumoniae KP715242 using central composite design. Biotechnol. Rep. 2015, 7, 128-134. [CrossRef] [PubMed]

23. Tamura, K.; Stecher, G.; Peterson, D.; Filipski, A.; Kumar, S. MEGA6: Molecular evolutionary genetics analyses version 6.0. Mol. Biol. Evol. 2013, 30, 2725-2729. [CrossRef] [PubMed]

24. Aguilar, C.N.; Augur, C.; Favela-Torres, E.; Viniegra-González, G. Induction and repression patterns of fungal tannase in solid-state and submerged cultures. Process Biochem. 2001, 36, 565-570. [CrossRef]

25. Sharma, S.; Bhat, T.K.; Dawra, R.K. A spectrophotometric method for assay of tannase using rhodanine. Anal. Biochem. 2000, 279, 85-89. [CrossRef] [PubMed] 
26. Pfaffl, M.W. A new mathematical model for relative quantification in real-time RT-PCR. Nucleic acids Res. 2001, 29, e45. [CrossRef]

27. Chi, Z.; Wang, X.X.; Ma, Z.C.; Buzdar, M.A.; Chi, Z.M. The unique role of siderophore in marine-derived Aureobasidium pullulans HN6. 2. Biometals 2012, 25, 219-230. [CrossRef] [PubMed]

28. Banerjee, A.; Jana, A.; Pati, B.R.; Mondal, K.C.; Mohapatra, P.K.D. Characterization of tannase protein sequences of bacteria and fungi: An in silico study. Protein J. 2012, 31, 306-327. [CrossRef] [PubMed]

29. Suzuki, K.; Hori, A.; Kawamoto, K.; Thangudu, R.R.; Ishida, T.; Igarashi, K.; Koseki, T. Crystal structure of a feruloyl esterase belonging to the tannase family: A disulfide bond near a catalytic triad. Proteins Struct. Funct. Bioinform. 2014, 82, 2857-2867. [CrossRef]

30. Jiménez, N.; Curiel, J.A.; Reverón, I.; de las Rivas, B.; Muñoz, R. Uncovering the Lactobacillus plantarum WCFS1 gallate decarboxylase involved in tannin degradation. Appl. Environ. Microbiol. 2013, 79, 4253-4263.

31. Huang, W.; Ni, J.; Borthwick, A.G. Biosynthesis of valonia tannin hydrolase and hydrolysis of valonia tannin to ellagic acid by Aspergillus SHL 6. Process Biochem. 2005, 40, 1245-1249. [CrossRef]

32. Jiménez, N.; Esteban-Torres, M.; Mancheño, J.M.; de las Rivas, B.; Muñoz, R. Tannin degradation by a novel tannase enzyme present in some Lactobacillus plantarum strains. Appl. Environ. Microbiol. 2014, 80, 2991-2997.

33. Banerjee, A.; Singha, K.; Soren, J.P.; Sen, A.; Mondal, K.C.; Mohapatra, P.K.D. Evolutionary study and sequence-structure relationship of fungal tannase and its subcellular localization through bioinformatics. Life Sci. Inf. Publ. 2017, 3, 7.

34. Aithal, M.; Belur, P.D. Enhancement of propyl gallate yield in nonaqueous medium using novel cell-associated tannase of Bacillus massiliensis. Prep. Biochem. Biotechnol. 2013, 43, 445-455. [CrossRef] [PubMed]

35. Purohit, J.S.; Dutta, J.R.; Nanda, R.K.; Banerjee, R. Strain improvement for tannase production from co-culture of Aspergillus foetidus and Rhizopus oryzae. Bioresour. Technol. 2006, 97, 795-801. [CrossRef] [PubMed]

(C) 2019 by the authors. Licensee MDPI, Basel, Switzerland. This article is an open access article distributed under the terms and conditions of the Creative Commons Attribution (CC BY) license (http://creativecommons.org/licenses/by/4.0/). 